\title{
ANTIFERROMAGNETIC RESONANCE IN COO/NIO MIXED CRYSTALS
}

\author{
G. GEIS, R. GEICK and C.R. BECKER \\ Physikalisches Institut, D-8700 Würzburg, Germany \\ V. WAGNER \\ ILL Grenoble, France
}

\begin{abstract}
We have studied the lowest magnetic excitation of $\mathrm{Ni}_{1-x} \mathrm{Co}_{x} \mathrm{O}$ mixed crystals for $0.94 \leqslant x \leqslant 1$. Together with previous results for $0.02 \leqslant x \leqslant 0.07$ and neutron data for $x=0.14$ and $x=0.30$, the results are discussed by means of a model, especially the variation of AFMR frequency and preferred spin direction with Co concentration $x$.
\end{abstract}

\section{Introduction}

Among the antiferromagnetic oxides with rocksalt structure, $\mathrm{NiO}$ is a pure spin system with singlet orbital ground state, and its spin waves are well described by a Heisenberg model [1]. In $\mathrm{CoO}$ however, the orbital angular momentum is not quenched but is $L_{\text {eff }}=1$ in the ${ }^{4} \Gamma_{4}$ ground state of $\mathrm{Co}^{2+}$. The magnetic excitations can therefore be partly collective and partly single ion in nature [1].

The magnetic excitations of $\mathrm{CoO}$ and $\mathrm{NiO}$ have been studied by various methods, especially the AFMR frequencies by far infrared spectroscopy [1]. In both materials, only one AFMR mode has been observed though two modes with distinct frequencies are expected in these easy plane antiferromagnets. In $\mathrm{NiO}$, the lower AFMR frequency is probably too small to be observed in zero magnetic field.

\section{Experimental results}

We have studied the antiferromagnetic resonance of $\mathrm{Co}_{x} \mathrm{Ni}_{1-x} \mathrm{O}$ mixed crystals for essentially two reasons. At first, the anisotropy field acting on the Co ions is mostly single ion anisotropy (orbital effect etc.) and is much larger than that of the $\mathrm{Ni}$ ions which originates mainly from magnetic dipole interactions. Secondly, the spins point along a $\langle 11 \overline{2}\rangle$ direction in $\mathrm{NiO}$ and, in $\mathrm{CoO}$, their preferred direction is away from $\langle 11 \overline{2}\rangle$ by $7^{\circ} 50^{\prime}$ towards $\langle 00 \overline{1}\rangle$ [2]. Apart from the shift of the AFMR frequencies from $36 \mathrm{~cm}^{-1}$ in $\mathrm{NiO}$ to $146 \mathrm{~cm}^{-1}$ in $\mathrm{CoO}$ due to the difference of the anisotropy fields (and the exchange fields), there must be a change of the preferred spin direction from $\mathrm{NiO}$ to $\mathrm{CoO}$ in the mixed crystal system. In addition to a previous investigation of AFMR for small Co concentrations $(0 \leqslant x \leqslant 0.07)$ [1], we have measured the frequency of the lowest excitation in $\mathrm{Ni}$ doped
$\mathrm{CoO}(0.94 \leqslant x \leqslant 1)$ in the far infrared at liquid helium temperatures. For higher $\mathrm{Co}$ - or $\mathrm{Ni}$ concentrations $(0.07 \leqslant x \leqslant 0.93)$, the AFMR lines are broadened too much to be observed. However, for $x=0.10,0.14$, and 0.30 the $q=0$ magnon energies are known from neutron inelastic scattering [3]. All experimental data are compiled in fig. 1. Only one AFMR mode has been observed in most cases as in pure $\mathrm{NiO}$ and

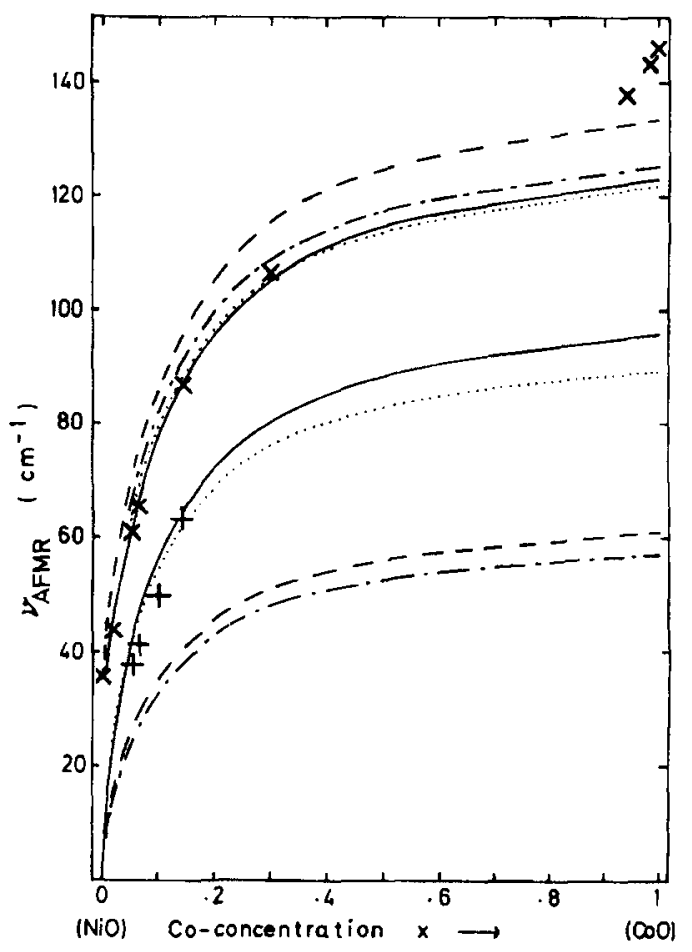

Fig. 1. AFMR frequencies of $\mathrm{Ni}_{1-x} \mathrm{Co}_{x} \mathrm{O}$ mixed crystals versus Co-concentration $x$, experimental values (upper: $x$, lower: + ) and values calculated by means of model $A$

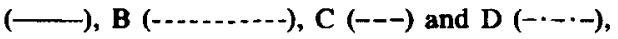

$\mathrm{CoO}$, except for small concentrations on the $\mathrm{Ni}$ rich side where two absorption lines have been found. 


\section{Model considerations}

The interpretation of the experimental data is based on a model which was described in detail previously [1]. The basic assumptions are

I. In the AFMR, all $\mathrm{Ni}$ ions and all Co ions in one of the two sublattices move in phase. They are treated as four quasi-sublattices with the appropriate statistical weight.

II. Each ion (Co or $\mathrm{Ni}$ ) is subject to an average exchange field arising from neighbouring $\mathrm{Co}$ and $\mathrm{Ni}$ ions (cf. eq. (1) in [1]).

III. For the anisotropy of the Ni ions (cf. eq. (2) and table 3 of [1]), only a trigonal term with constant parameter $T^{\mathrm{H}}$ is taken into account, neglecting the influence of the Co ions on $T^{\mathrm{H}}$ (dipole interactions!) and the small in-plane anisotropy $\left(U^{\mathrm{H}}=0\right)$.

IV. For the anisotropy of the Co ions, terms of trigonal, cubic and tetragonal symmetry are considered with parameters $T^{\mathrm{D}}, K^{\mathrm{D}}$, and $R^{\mathrm{D}}$, resp.

By minimizing the total energy (exchange plus anisotropy) with respect to rotations of the spins, the equilibrium directions were determined described by the angles $\Theta_{0}^{\mathrm{H}}(\mathrm{Ni})$ and $\Theta_{0}^{\mathrm{D}}$ (Co) between them and $\langle 11 \overline{2}\rangle$. Thus, our model allows for a rotation of the preferred direction between $\mathrm{NiO}$ and $\mathrm{CoO}$. For small oscillatory deviations from the equilibrium, the AFMR frequencies have been calculated. In contrast to [1], the approximation $\Theta_{0}^{\mathbf{H}}=\Theta_{0}^{\mathrm{D}}$ (strong exchange coupling!) and the approximations for small $x$ (cf. eq. (3) of [1]) have not been used, and the present results (cf. fig. 1 and table I) are correct for all $x$ within the model.

Table 1

Model parameters

\begin{tabular}{|c|c|c|c|c|c|}
\hline Model & \multicolumn{4}{|c|}{ Anisotropy parameters $\left(\mathrm{cm}^{-1}\right)$} & $\begin{array}{l}\text { Exchange parameters } \\
\left(\mathrm{cm}^{-1}\right)[1]\end{array}$ \\
\hline A & 0.25 & 10.90 & 22.10 & 0 & $J_{\mathrm{Ni}-\mathrm{Ni}}=71.33$ \\
\hline B & 0.25 & 9.50 & $\mathbf{0}$ & 10.40 & $J_{\mathrm{Ni}-\mathrm{Co}_{0}}=31.67$ \\
\hline $\mathrm{C}$ & 0.25 & 21.50 & 17.91 & $\mathbf{0}$ & $J_{\mathrm{CaCo}}=12.25$ \\
\hline D & 0.25 & 19.00 & 15.83 & 0 & $\left(S_{\mathrm{Ni}}=1, \quad S_{\mathrm{Co}}=1.7\right)$ \\
\hline
\end{tabular}

\section{Discussion}

For models A and B (cf. fig. 1 and table I), the parameters $T^{\mathrm{D}}$ and $K^{\mathrm{D}}$ or $R^{\mathrm{D}}$, resp., were chosen to obtain an optimum fit to the experimental data for $0 \leqslant x \leqslant 0.30\left(T^{\mathrm{H}}\right.$ is deter- mined by the AFMR of pure NiO). Then, the calculated preferred direction for $x=1(\mathrm{CoO})$ is $\Theta_{0}^{D} \approx 18^{\circ}$, i.e. more than the actual value. Generally, a better fit is obtained with a trigonal term $\left(T^{D}\right)$ and a cubic term $\left(K^{D}\right)$ instead of a tetragonal term for the Co ions. For models $\mathrm{C}$ and $\mathrm{D}, T^{\mathrm{D}}$ and $K^{\mathrm{D}}$ are balanced in such a way that $\Theta_{0}^{D}=9^{\circ}\left(\approx 7^{\circ} 50^{\prime}\right)$ for CoO. Now, the agreement between experimental and calculated data is poorer for $0 \leqslant x \leqslant 0.30$. For all models, we observe a smooth rotation of the preferred direction from $\Theta_{0}^{\mathrm{H}}=0(\mathrm{NiO})$ to $\Theta_{0}^{\mathrm{D}}$ in CoOCiO. This rotation takes place in the range $0 \leqslant x \leqslant 0.20$ associated with a drastic change of AFMR frequencies (cf. table II). The available data do not indicate a sudden flop from $\Theta_{0}=0$ in $\mathrm{NiO}$ to $7^{\circ} 50^{\prime}$ in $\mathrm{CoO}$ at a certain value of $x$. In all cases, the calculated frequencies are for $0.9 \leqslant x \leqslant 1$ (cf. fig. 1) considerably smaller than the experimental values. We believe that our data include only single Co-ion effects, and that there are additional collective contributions to the anisotropy. With an increased trigonal term $T^{\mathrm{D}}=27 \mathrm{~cm}^{-1}$ and with $K^{\mathrm{D}}=20 \mathrm{~cm}^{-1}$ (cf. model A), we obtain $\Theta_{0}=8^{\circ}$ and $\nu_{\mathrm{CoO}}=147 \mathrm{~cm}^{-1}$ in agreement with existing theories $[4,5]$. The model results show also the frequency of the lower AFMR-mode for the whole concentration range which has been observed only for $0.05 \leqslant$ $x \leqslant 0.14$.

Table II

Calculated equilibrium directions of $\mathrm{Ni}\left(\Theta_{0}^{\mathrm{H}}\right)$ and $\mathrm{Co}\left(\Theta_{0}^{\mathrm{D}}\right)$ ions.

\begin{tabular}{lcccccccc}
\hline Model & \multicolumn{7}{c}{ Co-concentration $x$} \\
& & 0 & 0.04 & 0.1 & 0.2 & 0.4 & 0.6 & 1 \\
\hline A & $\Theta_{0}^{\mathrm{H}}$ & $0^{\circ}$ & $13.8^{\circ}$ & $17.0^{\circ}$ & $18.3^{\circ}$ & $19.0^{\circ}$ & $19.3^{\circ}$ & - \\
& $\Theta_{0}^{\mathrm{D}}$ & - & $14.2^{\circ}$ & $17.2^{\circ}$ & $18.4^{\circ}$ & $19.1^{\circ}$ & $19.3^{\circ}$ & $19.5^{\circ}$ \\
B & $\Theta_{0}^{\mathrm{H}}$ & $0^{\circ}$ & $13.4^{\circ}$ & $16.2^{\circ}$ & $17.4^{\circ}$ & $18.1^{\circ}$ & $18.3^{\circ}$ & - \\
& $\Theta_{0}^{\mathrm{D}}$ & - & $13.8^{\circ}$ & $16.4^{\circ}$ & $17.5^{\circ}$ & $18.1^{\circ}$ & $18.4^{\circ}$ & $18.5^{\circ}$ \\
$\mathrm{C}$ & $\Theta_{0}^{\mathrm{H}}$ & $0^{\circ}$ & $6.8^{\circ}$ & $8.1^{\circ}$ & $8.6^{\circ}$ & $8.9^{\circ}$ & $9.0^{\circ}$ & - \\
& $\Theta_{\mathrm{O}}^{\mathrm{D}}$ & - & $7.0^{\circ}$ & $8.2^{\circ}$ & $8.6^{\circ}$ & $8.9^{\circ}$ & $9.0^{\circ}$ & $9.0^{\circ}$ \\
\hline
\end{tabular}

\section{References}

[1] C.R. Becker, Ph. Lau, R. Geick and V. Wagner, Phys. stat. sol. (b) 67 (1975) 653, and references therein.

[2] M.D. Rechtin and B.L. Averbach, Phys. Rev. B6 (1972) 4294.

[3] V. Wagner, D. Tocchetti and B. Hennion, Verh. DPG (VI) 9 (1974) 645.

[4] J. Kanamori, Progr. theor. Phys. (Kyoto) 17 (1957) 177, 197.

[5] O. Nakanishi and T. Yamada, J. Phys. Soc. Japan 36 (1974) 1315 